\title{
CD1a+ and CD83+ Langerhans cells are reduced in lower lip squamous cell carcinoma
}

Juliane Oliveira Gomes ${ }^{1}$, Marianne de Vasconcelos Carvalho ${ }^{2}$, Felipe Paiva Fonseca ${ }^{1}$,

Rogério Oliveira Gondak ${ }^{3}$,Márcio Ajudarte Lopes ${ }^{1}$, Pablo Agustin Vargas ${ }^{1,4}$

\footnotetext{
${ }^{1}$ Department of Oral Diagnosis, University of Campinas, Piracicaba, Brazil;

${ }^{2}$ Department of Dentistry, University of Pernambuco, Recife, Brazil;

${ }^{3}$ Department of Pathology, Federal University of Santa Catarina, Florian opolis, Brazil;

${ }^{4}$ Department of Oral Pathology and Oral Biology, Faculty of Health Sciences, School of Dentistry, University of Pretoria, Pretoria, South Africa
}

\begin{abstract}
:
Background: Actinic cheilitis (AC) is a potentially malignant lesion diagnosed in the lip of patients chronically exposed to the sun that may give rise to a fully invasive lower lip squamous cell carcinoma (LLSCC). It is known that ultraviolet radiation causes dendritic cells (DCS) depletion in the epidermis, but the role of this cellular population in lip cancer progression remains uncertain. Therefore, this study investigated the distribution of DCs in normal, dysplastic and neoplastic tissues of the lower lip.
\end{abstract}

Methods: Thirteen cases of lower lip mucocele, 42 of ACs and 21 of LLSCC were retrieved and original diagnoses confirmed by two oral pathologists, who further classified ACs as low- and high-risk lesions. Immunoreactions against CD1a and CD83 identified immature and mature DCs, respectively.

Results: Immature CD1a+ Langerhans cells (LCs) were significantly decreased in LLSCC when compared to morphologically normal $(P<0.009)$ and dysplastic epitheliums $(P<0.003)$, whereas mature CD83+ LCs were significantly decreased in LLSCC when compared to normal epithelium ( $P=0.038)$. There was no significant difference between low- and high-risk ACs regarding CD1a+ and CD83+ LCs $(P>0.05)$, but ACs demonstrated a lower concentration of CD1a+ LCs than normal epithelium $(P<0.009)$. There was no significant difference in the distribution of CD1a+ and CD83+ interstitial dendritic cells (IDCs) in the connective tissue among the studied groups $(P>0.05)$.

Conclusion: These results suggest that depletion of epithelial LCs, but not IDCs in the connective tissue, would represent an important step for lip cancer development. 


\section{Introduction}

Actinic cheilitis (AC) is a potentially malignant lesion that most commonly affects the lower lip of light-skinned individuals whose occupational activities are related to chronic sun exposure as a result of molecular and genetic alterations in response to long-term ultraviolet radiation [1-3]. Histological changes frequently include different grades of epithelial dysplasia that in advanced stage, lesions may achieve fully developed invasive carcinomas. Lower lip squamous cell carcinoma (LLSCC) normally has a favorable prognosis with an expected 10 -year survival rate exceeding $90 \%$ in stage I disease, but significantly decreasing in more advanced stage tumors [4]. Although distant dissemination is found in approximately $1 \%$ of the patients $[4,5]$, lymph node metastases seem to occur in a variable rate ranging from as low as $3 \%$ up to $22 \%$ of the cases [4, 6-9].

Langerhans cells (LCS) are bone marrow-derived dendritic cells (DCs) that represent 2-3\% of the entire cell population of normal skin and mucosal epitheliums [10]. LCs are responsible for taking antigens found in the squamous epithelium to regional draining lymph nodes. Upon transfer to connective tissue (interstitial dendritic cells - IDCS) and posterior arrival in the paracortex of lymph nodes, the antigen-laden LCs transform into interdigitating DCs and they present antigen to naive T lymphocytes [11-13]. DCs are speculated to be involved in immunosurveillance against neoantigens associated with malignant transformation by transporting these antigens to local lymph node and presenting them to specific T cells [12].

A relationship between DCs and prognosis of patients affected by different cancers has been published $[12,14]$, raising the question of whether DCs would have a role in the immunosurveillance against cancer. However, the presence and significance of DCs in the LLSCC development remain uncertain. Thus, the aim of this study was to compare the presence and distribution of LCs (in the epithelial compartment) and IDCs (in the connective tissue compartment) in morphologically normal lower lip mucosa, low- and high-risk ACs, and LLSCC.

\section{Material and methods}

In a 10-year period from January 2000 to December 2009, 42 cases of AC and 21 of LLSCC were retrospectively retrieved from the files of the Department of Oral Diagnosis of the University of Campinas (Piracicaba Dental School, Brazil). Clinical information regarding age, gender, presence of pain, and ulceration was collected from patients' records. For LLSCC cases with follow-up data available, presence of recurrences was also assessed. The diagnoses were confirmed by two independent oral pathologists by reviewing the original $5-\mu m$ histological sections stained with hematoxylin and eosin (H\&E), and cases of ACs were classified as low risk and high risk according to binary system of grading oral epithelial dysplasia described by Kujan et al. [15]. Thirteen cases of morphologically normal epithelium and connective tissue adjacent to lower lip mucoceles were used as control group.

Immunohistochemistry was performed according to the methods of Andrade et al. [16]. Briefly, the reactions were carried out in 3- $\mu \mathrm{m}$ sections of the original formalin-fixed, paraffin- 
embedded tissues that were de-waxed with xylene and hydrated in a descendent ethanol series. Antigen retrieval was performed and the endogenous peroxidase activity was blocked using $10 \%$ hydrogen peroxide. After washing in PBS buffer $(\mathrm{pH} 7.4)$, slides were incubated overnight with the primary antibodies anti-CD83 (Clone 1H4b, 1:20; Novocastra, Newcastle upon Tyne, UK) for reacting against mature DCs and anti-CD1a (Clone 010, dilution 1:80; DAKO Co., Carpentia, CA, USA) for evidencing the presence of immature DCs. The cellular morphology of DCs was also evaluated for considering positivity. All slides were subsequently exposed to avidin-biotin complex and horseradish peroxidase reagents (LSAB Kit; DakoCytomation, Carpinteria, CA, USA) and diaminobenzidin tetrahydrochloride (DAB; Sigma, St. Louis, MO, USA), and subsequently counterstained with Carazzi hematoxylin. Normal skin was used as positive control, whereas the negative control was obtained by omitting the primary specific antibody.

Quantification of DCs was jointly performed by two oral pathologists following the methods previously described $[10,13]$ using a light microscope (Nikon, Tokyo, Japan) with a 40x eyepiece and a $8 \times$ reticulated square (Reichert, Vienna, Austria) with a length of $150 \mu \mathrm{m}$ and an area 22 $500 \mathrm{\mu m}^{2}$. Positive DCs were counted for each antibody in seven sequential fields and quantified for each case, comprising an area of $1 \mathrm{~mm}^{2}$ of analyzed tissue in each region. The results were expressed as positive cells per area (mean cells $/ \mathrm{mm}^{2}$ ). Cells identified in the epithelium were interpreted as LCs (CD1a+ immature LC and CD83+ mature LC) and those found in the subepithelial connective tissue were interpreted as IDCs (CD1a+ immature IDC and CD83+ mature IDC) [13].

Kolmogorov-Smirnov test was applied to analyze data distributions. The values were expressed as mean \pm standard deviation (SD), and the comparison between the groups was performed by analysis of variance (ANOVA), followed by Tukey and Bonferroni post hoc tests. A $P$-value of $<0.05$ was regarded as statistically significant. Correlations were tested using Pearson's test. The statistical software SPSS version 18.0 was used for the analyses. This study was approved by the Ethics Committee of the Piracicaba Dental School, University of Campinas (protocol 118/2011).

\section{Results}

Clinical data of the patients are shown in Table 1. Females predominated in the control group, whereas a male preponderance was found in the other groups analyzed. Patients with LLSCC exhibited a higher mean age (68.9 years) than patients with mucocele (19.2 years) and AC (low risk 59.5 years and high risk 64 years). Ulceration was present in 1 case of low-risk $A C$ (4.7\%), 13 cases of high-risk AC (61.9\%), and 13 cases of LLSCC (61.9\%; Fig. 1), whereas pain was reported by two patients with low-risk AC (9.5\%), three patients with high-risk $A C(14.2 \%)$, and nine patients with LLSCC (42.8\%). Follow-up data was available for 12 patients (57\%) affected by LLSCC (mean time of 34.4 months; range 1-91 months), with three patients developing recurrences. No patient died during the follow-up period. 
Table 1. Clinicopathological features of normal oral mucosa, AC with different grades of epithelial dysplasia and LLSCC

\begin{tabular}{|c|c|c|c|c|c|}
\hline Groups & Age (years) & Gender (M:F) & Ulceration & Pain & Recurrence \\
\hline \multicolumn{6}{|c|}{ 1. AC, actinic cheilitis; LLSCC, lower lip squamous cell carcinoma. } \\
\hline Control group $(n=13)$ & $19.2 \pm 12.6$ & $6: 7$ & $0(0.0 \%)$ & $0(0.0 \%)$ & - \\
\hline AC with low-grade dysplasia $(n=21)$ & $59.5 \pm 10.9$ & $20: 1$ & $1(4.7 \%)$ & $2(9.5 \%)$ & - \\
\hline AC with high-grade dysplasia $(n=21)$ & $64 \pm 9.4$ & $13: 8$ & $13(61.9 \%)$ & $3(14.2 \%)$ & - \\
\hline $\operatorname{LLSCC}(n=21)$ & $68.9 \pm 10.9$ & $15: 6$ & $13(61.9 \%)$ & $9(42.8 \%)$ & $3(14.2 \%)$ \\
\hline
\end{tabular}
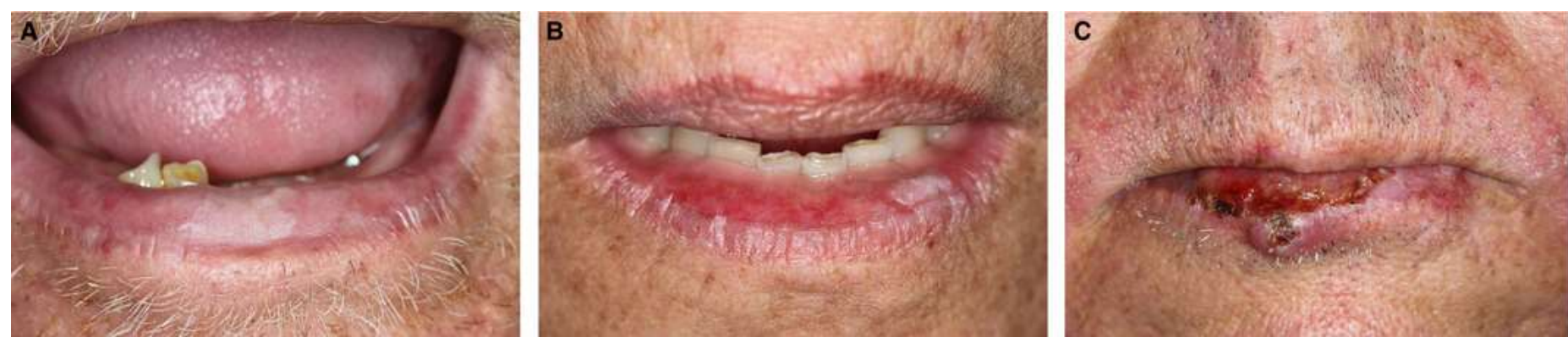

Figure 1. Representative clinical images of three patients affected by actinic cheilitis (AC) and lower lip squamous cell carcinoma (LLSCC) that were included in the current sample. (A) AC microscopically diagnosed as low-risk dysplasia, clinically demonstrating a whitish plaque in the central area of the lower lip. (B) AC microscopically diagnosed as high-risk dysplasia with a whitish plaque in the left side of the lower lip. (C) LLSCC with extensive ulcerative areas.

Microscopically, in the mucocele/control group, morphologically normal epithelium and connective tissue (with no or mild inflammatory infiltrate) were used for analysis, and no evidence of epithelial dysplasia or solar elastosis was found. Cases of ACs demonstrated areas of acanthosis and hyperkeratosis, or regions of atrophic epithelium. Mild to moderate chronic inflammatory infiltrate was present in all cases. Degenerative collagenous fibers (solar elastosis) in the connective tissue were present in all cases of ACs and LLSCC. Cases classified as ACs with low-risk epithelial dysplasia demonstrated mild cellular and architectural alterations mostly confined to basal and parabasal layers of the epithelium, whereas those included in the highrisk group exhibited an increased amount of cellular atipia in upper layers of the epithelium. All cases of LLSCC consisted of well-differentiated carcinomas with keratin pearls formation, cellular pleomorphism and atypical mitotic figures, infiltrating adjacent normal structures (Fig. 2). 


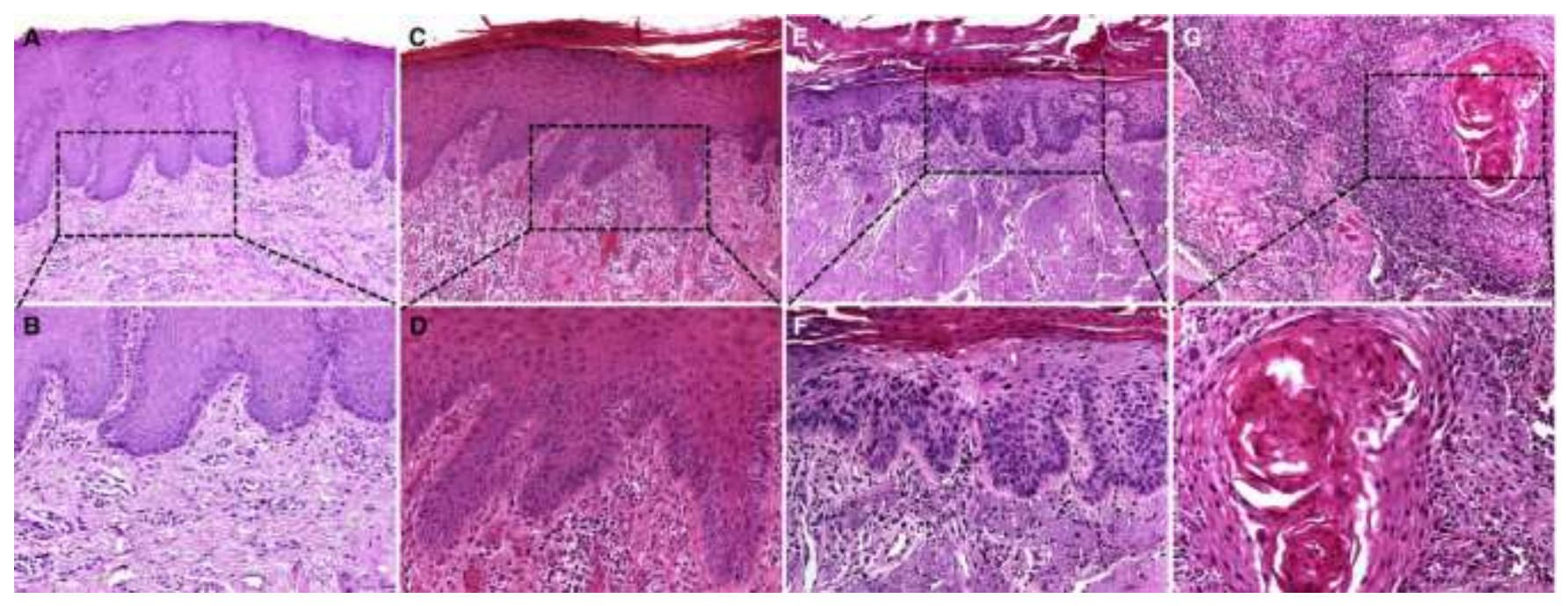

Figure 2. Histopathological features of control group (mucocele), actinic cheilitis (AC), and lower lip squamous cell carcinoma (LLSCC). (A) Histologically normal oral epithelium without dysplastic alterations (hematoxylin and eosin - H\&E; 100x). (B) Higher magnification of normal epithelium group demonstrating connective tissue devoid of solar elastosis (H\&E; 200x). (C) Microscopic features of AC with low-grade dysplasia demonstrating mild cytological atypia predominantly located in basal and parabasal layers of the epithelium (H\&E; 100x). (D) Higher magnification demonstrating mild epithelial dysplasia (H\&E; 200x). (E) Dysplastic cytological and architectural features are more frequent in cases classified as AC with high-grade dysplasia (H\&E; 100x). (F) Higher magnification illustrating the severe epithelial dysplasia in an atrophic epithelium. Note the strong solar elastosis in the connective tissue (H\&E; 200x). (G) Well-differentiated LLSCC with infiltrative features, cellular atipia, and keratin pearls formation (H\&E; 100x). (H) Higher magnification of keratin pearl formation with few mitoses and cellular pleomorphism.

Dendritic cells were identified as ramified cells in normal, dysplastic, and neoplastic epitheliums, and in the connective tissue of the studied samples (Fig. 3). Regarding distribution of LCs in the epithelial compartment (Fig. 4), immature CD1a+ cells were significantly decreased in LLSCC when compared to both normal oral epithelium $(P<0.009)$ and AC groups $(P<0.003)$, as well as in ACs when compared to normal oral epithelium $(P<0.009)$. On the other hand, mature CD83+ LCs were only significantly reduced in LLSCC compared to normal oral epithelium $(P=0.038)$. There was no difference in the LCs density between low-risk and high-risk ACs using both CD1a and CD83 markers ( $P>0.05$; Table 2$)$. 

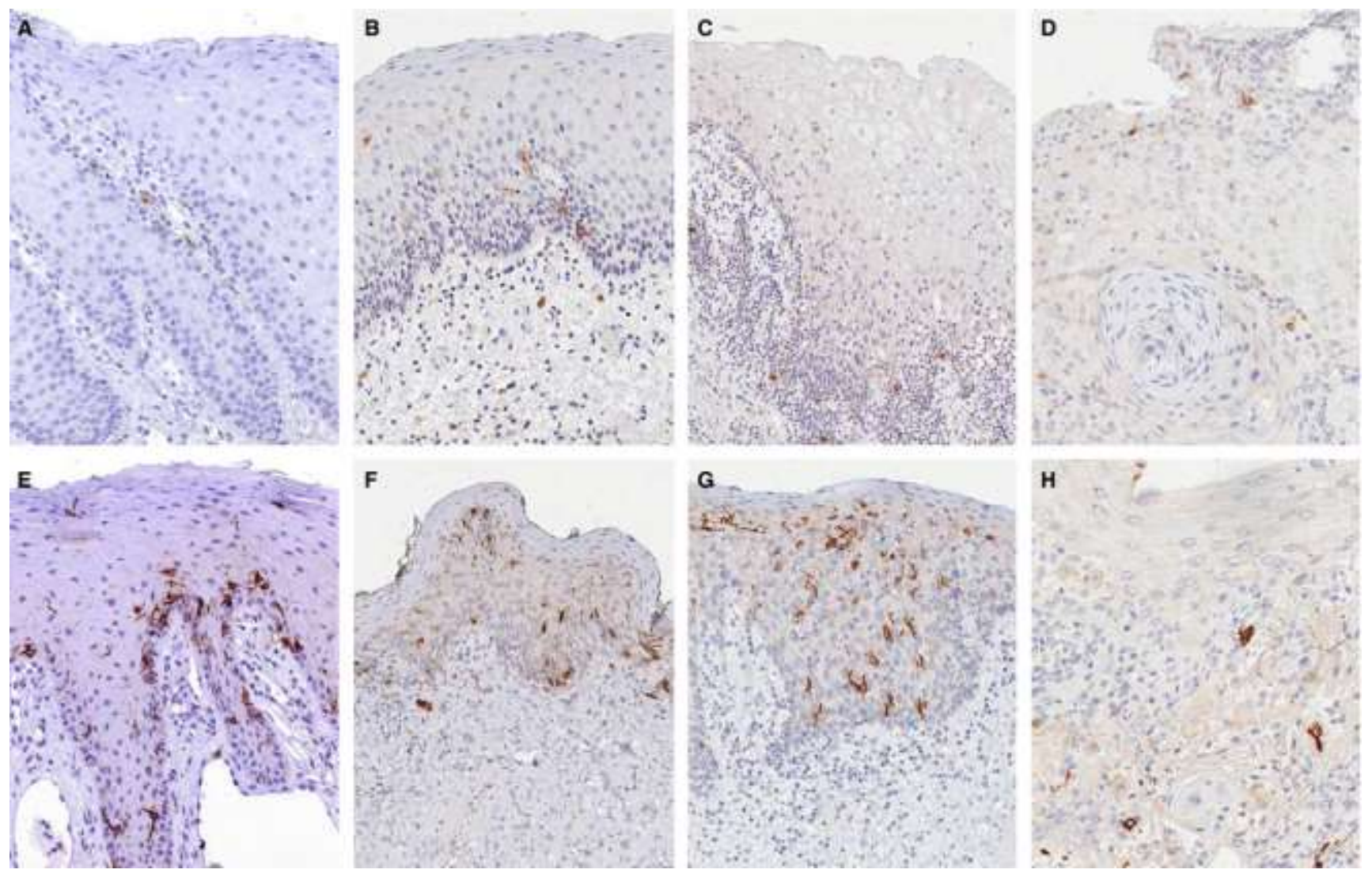

Figure 3. Immunohistochemical detection of mature and immature Langerhans cells (LCs) in the control group (mucocele), actinic cheilitis (AC), and lower lip squamous cell carcinoma (LLSCC) using CD83 and CD1a antibodies, respectively. (A) Immunohistochemical expression of CD83+ cells in the epithelium and the subepithelial connective tissue of the control group (mucocele) (streptavidin-biotin; 200x). (B) AC with low-grade dysplasia group (streptavidin-biotin; 200x). (C) AC with high-grade dysplasia (streptavidin-biotin; 100x). (D) LLSCC (streptavidin-biotin; 200x). (E) CD1a+ cells in the control group (mucocele) (streptavidin-biotin; 200x). (F) AC with low-grade dysplasia (streptavidin-biotin; 100x). (G) AC with high-grade dysplasia (streptavidin-biotin; 100x). (H) LLSCC (streptavidin-biotin; 200x). There was a remarkable decrease of immature (CD1a+) and mature (CD83+) LCs in the epithelium of LLSCC group, but no significant difference in the presence of CD1a+ and CD83+ interstitial dendritic cells in the subepithelial connective tissue among the groups investigated. 

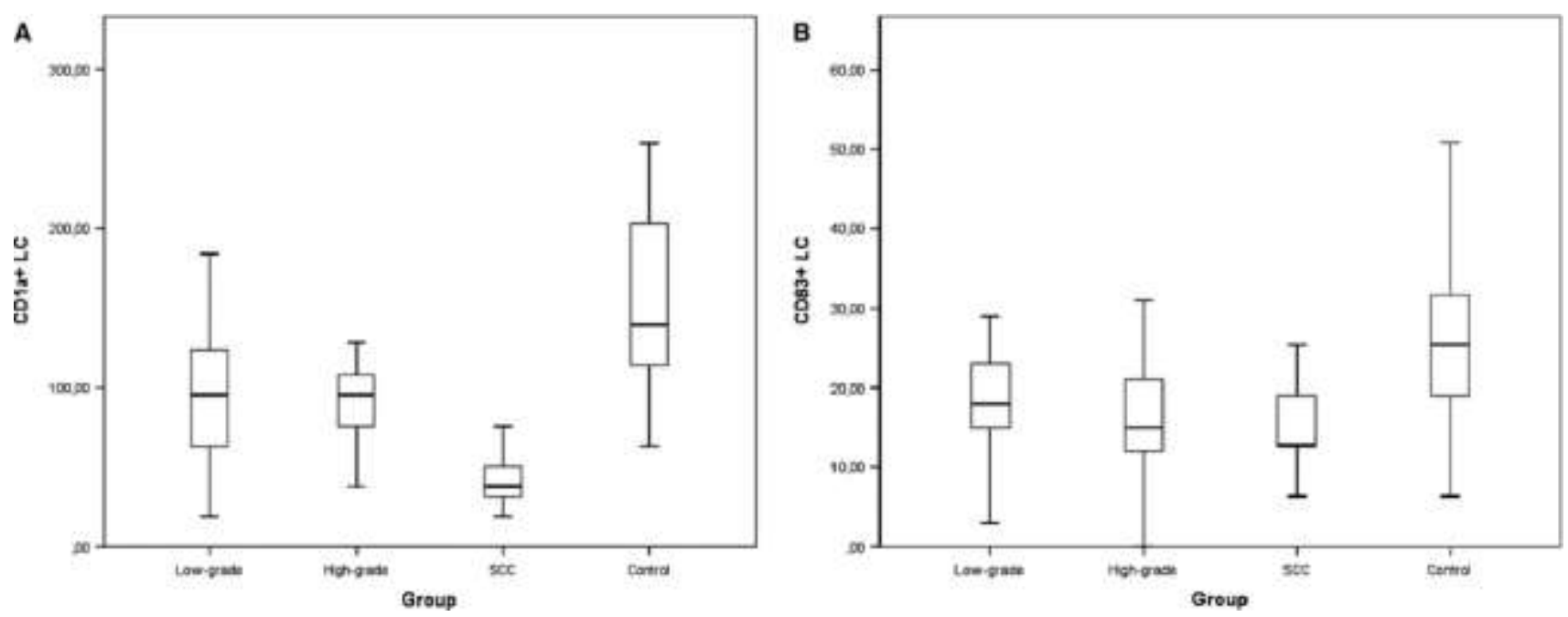

Figure 4. Distribution of immature (CD1a+) and mature (CD83+) Langerhans cells (LCS) in the epithelial compartment of the control group, low-risk actinic cheilitis (AC), high-grade AC, and lower lip squamous cell carcinoma (LLSCC). Immature LCs were significantly decreased in LLSCC when compared to control group and AC groups, and between AC groups and control group, whereas mature LCs were significantly decreased in LLSCC when compared to control group.

Table 2. Quantification of CD1a+ and CD83+ cells (mean of cells $/ \mathrm{mm}^{2} \pm \mathrm{SD}$ ) in the epithelium and connective tissue of all groups

\begin{tabular}{||l|l|l|l|l||c||}
\hline Antibody & Compartment & Control group & Low-risk AC & High-risk AC & \multicolumn{1}{|c|}{ LLSCC } \\
\hline \multirow{3}{*}{ CD1a } & Epithelium (LC) & $154.82 \pm 56.40^{\mathrm{a}}$ & $97.91 \pm 52.32^{\mathrm{b}}$ & $101.68 \pm 41.14^{\mathrm{c}}$ & $44.44 \pm 20.65^{\mathrm{d}}$ \\
\cline { 2 - 6 } & Connective tissue (IDC) & $27.35 \pm 18.21$ & $25.58 \pm 21.62$ & $37.56 \pm 32.65$ & $21.16 \pm 12.48$ \\
\hline \hline \multirow{2}{*}{ CD83 } & Epithelium (LC) & $27.35 \pm 18.21^{\mathrm{e}}$ & $18.52 \pm 6.64$ & $18.21 \pm 11.95$ & $15.14 \pm 6.62^{\mathrm{f}}$ \\
\hline
\end{tabular}

AC, actinic cheilitis; LLSCC, lower lip squamous cell carcinoma; LC, Langerhans cell; IDC, interdigitating dendritic cell.

Statistically significant differences were obtained with the following comparisons: $a$ vs. b, $c$ and $d(P<0.009)$; $d$ vs. b and $\mathrm{c}(P<0.003)$, e vs. $f(P=0.038)$.

Considering IDC density in the subepithelial connective tissue (Fig. 5), although LLSCC cases have also demonstrated a lower density of immature (CD1a+) and mature (CD83+) cells, there was no statistically significant difference among the groups investigated $(P>0.05$; Table 2$)$. Finally, there were no significant correlations between LC and IDC densities with 
clinicopathological data (age, gender, ulceration, pain, and recurrences; $P>0.05$ ), and all groups showed normal distribution according to Kolmogorov-Smirnov test $(P>0.08)$.
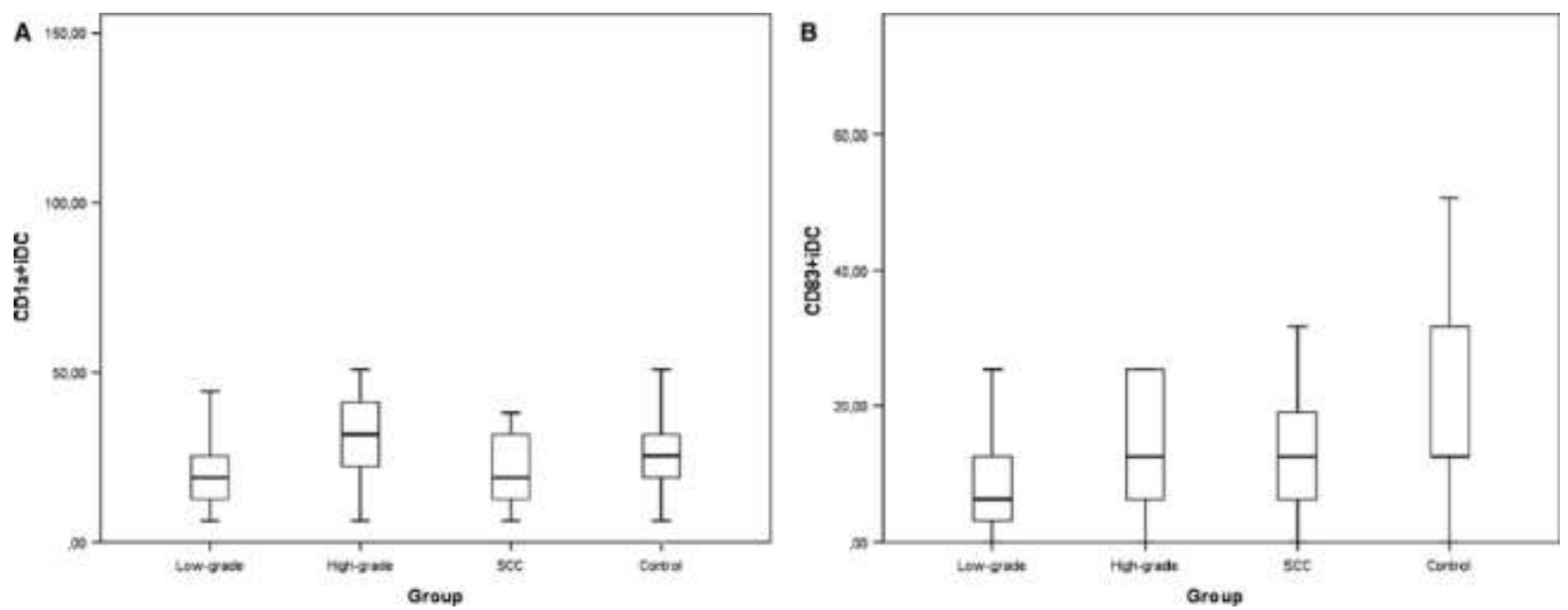

Figure 5. Distribution of immature $(\mathrm{CD} 1 \mathrm{a}+)$ and mature $(\mathrm{CD} 83+)$ interstitial dendritic cells (IDCs) in the connective tissue compartment of the control group, low-risk AC, high-grade AC, and lower lip squamous cell carcinoma (LLSCC). Although LLSCC demonstrated a lower density of IDCs (mature and immature), there was no significant difference among the groups analyzed.

\section{Discussion}

Lower lip squamous cell carcinoma has an elevated prevalence in white population chronically exposed to ultraviolet radiation [17], and AC is the most common potentially malignant disorder that precedes LLSCC, present in approximately 95\% of the cases [18]. Therefore, investigating cellular alterations that would favor malignant transformation of a previous dysplastic condition could provide new insights to better understand this process, and the innate immunoregulatory mechanisms are believed to play an important role in the recognition and destruction of altered dysplastic/neoplastic cells. Hence, in the current study we have demonstrated a significant decrease in the number of mature (CD83+) and immature (CD1a+) epithelial LCs (but not of IDCs in the subepithelial connective tissue) in patients affected by LLSCC, suggesting that an imbalance in the cellular immunosurvailence is an important early step for lower lip cancer development.

Dendritic cells are known to play a central role in the regulation of both innate and adaptive immunologic responses. Recent studies have suggested that the loss of DCs would correlate with tumor progression, lower survival rate, and poor prognosis for patients affected by different human neoplasms, possibly as a consequence of a deficit in the host immune response to tumor growth and a cytotoxic T lymphocytes impairment $[12,19]$. This was shown to be true for patients affected by colorectal [20, 21], gastric [22, 23], pancreatic [24], breast [25], and other human cancers, as well as in experimental animal models [26, 27]. Because of these clinically relevant findings, some DC-based cancer immunotherapies have been developed, but a meaningful clinical response remains to be achieved [28]. 
A large number of antibodies targeting DC markers are currently available, including those recognizing proteins like S100, CD1a, CD83, CD207, CD208, CD80, CD11c, CD34, CD86, HLA-DR, and others with different degrees of specificity and sensibility, but the incomplete knowledge of the biological significance of this growing number of markers impairs the determination of their clinical and prognostic relevance. CD1a is widely accepted as a marker of immature DCs, whereas CD83 is believed to be a determinant of mature cells [19]. Because the biological properties of these proteins in DC context have been well characterized and both have been previously investigated in different neoplasms, we opted for using CD1a and CD83 to identify immature and mature DCs, respectively.

Different authors investigated the importance of DCs for oral squamous cell carcinoma (OSCC) progression. Öhman et al. [11] evaluated the presence of CD1a+ LCs in oral leukoplakia and OSCC, demonstrating an increase in the presence of these cells toward the neoplastic phenotype, whereas van Heerden et al. [29] could not demonstrate any significant association between OSCC grade of differentiation and LC density. O'Donell et al. [30] claimed that the inability to induce an effective immune response would be more important for oral cancer development than the lack of DCs, what was further supported by Kikuchi et al. [31] that demonstrated that secreted VEGF inhibited the differentiation of CD1a+ DCs and increased the levels of dysfunctional CD83+ cells. Hence, we believe that the complex interaction of different DC subtypes with each human malignant neoplasm and the action of different etiologic agents involved with lip cancer and intra-oral cancer onset might explain the divergent results obtained in the current research where both mature and immature LCs were decreased in LLSCC when compared to normal epithelium, suggesting that depletion of LCs would be more relevant to the development of LLSCC than to OSCC. However, we could not obtain any significant correlation between the expression of LCs or IDCs with clinicopathological parameters in this study, possibly because of the relative small sample of LLSCC with available follow-up data.

It was demonstrated that exposure to UV radiation results in different degrees of change in LCs density and morphology, which would impair the function of these cells and induce changes in the immune response [32-35]. Thus, considering that prolonged exposure to UV rays is the main etiological factor for the development of LLSCC, it is speculated that the decrease in the amount of DCs, more specifically the epithelial LCs, in the irradiated tissue could be directly related to the deleterious effects of this radiation, impairing the immune response and assisting neoplastic growth [36]. On the other hand, Dumay et al. [37] demonstrated that the deleterious effects of UV rays on epithelial LCs can be partially prevented by application of sunscreen with protective factors, suggesting that filtering longer wavelengths UV absorption would promote a more favorable immune protection.

Analyzing the distribution of CD1a+ cells in ACs and normal lip epithelium, Araújo et al. [38] described an important increase in the number of LCs in the dysplastic cases, what is in contrast to the current findings where AC demonstrated a significant reduction of immature CD1a+ LCs when compared to normal epithelium, although no significant reduction was found regarding CD83+ LCs. This divergence is difficult to explain, but it is important to highlight that considering 
the difficulty and subjectivity related to histological grading of epithelial dysplasia, different from Araújo et al. [38], the current study adopted the binary classification proposed by Kujan et al. [15] that classifies oral dysplastic lesions as low risk and high risk, decreasing the variability of the histological criteria proposed by the WHO $[39,40]$.

Despite the disruptions caused by UV radiation in the distribution of immature and mature LCS studied in the current sample, it was not observed a significant difference in the population of mature and immature IDCs among normal, dysplastic, and neoplastic lower lip tissues. This could be attributed to the fact that IDCs are present in the lamina propria instead of in the overlying epithelium, possibly becoming less susceptible to the effects of UV rays.

In conclusion, although the malignant neoplastic transformation represents a multifactorial process, by demonstrating a significant depletion of LCs in the epithelium of LLSCC compared to normal lip epithelium and ACs, with no significant decrease of IDCs in the subepithelial connective tissue, we suggest that the suppression of CD1a+ and CD83+ LCs due to UV radiation could represent one of the primary steps for the development of lip cancer, demonstrating the importance of these cells in anti-LLSCC immunity.

\section{References}

1. Nico MM, Rivitti EA, Lourenço SV. Actinic cheilitis: histologic study of entire vermilion and comparison with previous biopsy. J Cutan Pathol 2007; 34: 309-14.

2. Piñera-Marques K, Lorenço SV, Silva LF, Sotto MN, Carneiro PC. Actinic lesions in fishermen's lower lip: clinical, cytopathological and histopathologic analysis. Clinics 2010; 65: 363-7.

3. da Silva Peixoto. J, Lourenço S, Nico M, Silva FH, Martins MT, Costa-Neves A. Expression of laminin-5 and integrins in actinic cheilitis and superficially invasive squamous cell carcinomas of the lip. Pathol Res Pract 2012; 208: 598-603.

4. Salihu S, Güven O, Gllareva E, Prekazi M, Salihu L. A clinical study on survival rate of patients with squamous cell carcinoma of the lower lip in Kosovo. J Craniomaxillofac Surg 2014; 42: 1773-7.

5. Santos HBP, Silva ALO, Cavalcante LHA, Alves PM, Godoy GP, Nonaka CFW. Histopathological grading systems and their relationship with clinical parameters in lower lip squamous cell carcinoma. Int J Oral Maxillofac Surg 2014; 43: 539-45.

6. Bilkay U, Kerem H, Ozek C. Management of lower lip cancer: a retrospective analysis of 118 patients and review of the literature. Ann Plast Surg 2003; 51: 43-50.

7. Rodolico V, Barresia E, Lorenzo R, et al. Lymph node metastasis in lower lip squamous cell carcinoma in relation to tumour size, histologic variables and p27Kip1 protein expression. Oral Oncol 2004; 40: 928.

8. Hasson O. Squamous cell carcinoma of the lower lip. J Oral Maxillofac Surg 2008; 66: 1259-62. 
9. Gutiérrez-Pascual M, Vicente-Martín FJ, Fernández-Álvarez JG, Martín-López R, Pinedo-Moraleda F, López-Estebaranz JL. Squamous cell carcinoma of the lip. A retrospective study of 146 patients. J Eur Acad Dermatol Venereol 2012; 26: 1116-21.

10. Gondak RO, Alves DB, Silva LF, Mauad T, Vargas PA. Depletion of Langerhans cells in the tongue from patients with advanced-stage acquired immune deficiency syndrome: relation to opportunistic infections. Histopathology 2012; 60: 497-503.

11. Öhman J, Magnusson B, Telemo E, Jontell M, Hasséus B. Langerhans cells and T cells sense cell dysplasia in oral leukoplakias and oral squamous cell carcinomas - evidence for immunosurveillance. Scand J Immunol 2012; 76: 39-48.

12. Esteban F, Ruiz-Cabello F, Gonzalez-Moles MA, Lopez-Gonzalez MA, Funez R, Redondo M. Clinical significance of Langerhans cells in squamous cell carcinoma of the larynx. J Oncol 2012; 2012: 753296300.

13. Gondak RO, Mauad T, Almeida OP, Vargas PA. Reduced number of CD1a+ and CD83+ interstitial dendritic cells in herpetic lesions (HSV-1+) of the tongue in patients with advanced-stage AIDS. Histopathology 2013; 63: 595-8.

14. La Rocca G, Anzalone R, Bucchieri F, Farina F, Cappello F, Zummo G. Review CD1a and antitumour immune response. Immunol Lett 2004; 95: 1-4.

15. Kujan O, Oliver RJ, Khattab A, Roberts SA, Thakker N, Sloan P. Evaluation of a new binary system of grading oral epithelial dysplasia for prediction of malignant transformation. Oral Oncol 2006; 42: 98793.

16. Andrade BAB, León JE, Carlos R, Delgado-Azañero W, Mosqueda-Taylor A, Almeida OP. Immunohistochemical expression of p16, p21, p27 and cyclin D1 in oral nevi and melanoma. Head Neck Pathol 2012; 6: 297-304.

17. Sugerman PB, Savage NW. Oral cancer in Australia: 1983-1996. Aust Dent J 2002; 47: 45-56.

18. Ariotti C, Wagner VP, Salvadori G, et al. VEGFR1 and VEGFR2 in lip carcinogenesis and its association with microvessel density. Tumour Biol 2015; 36: 7285-92.

19. Perez L, Shurin MR, Collins B, Kogan D, Tourkova IL, Shurin GV. Comparative analysis of CD1a, S-100, CD83, and CD11c human dendritic cells in normal, premalignant, and malignant tissues. Histol Histopathol 2005; 20: 1165-72.

20. Ambe K, Mori M, Enjoji M. S-100 protein-positive dendritic cells in colorectal adenocarcinomas. Distribution and relation to the clinical prognosis. Cancer 1989; 63: 496-503.

21. Gulubova MV, Ananiev JR, Vlaykova TI, Yovchev Y, Tsoneva V, Manolova IM. Role of dendritic cells in progression and clinical outcome of colon cancer. Int J Colorectal Dis 2012; 27: 159-69. 
22. Hu M, Li K, Maskey N, et al. Decreased intratumoral Foxp3 Tregs and increased dendritic cell density by neoadjuvant chemotherapy associated with favorable prognosis in advanced gastric cancer. Int J Clin Exp Pathol 2014; 7: 4685-94.

23. Amoueian S, Attaranzadeh A, Montazer M. Intratumoral CD68-, CD117-, CD56-, and CD1a-positive immune cells and the survival of Iranian patients with non-metastatic intestinal-type gastric carcinoma. Pathol Res Pract 2015; 211: 326-31.

24. Dallal RM, Christakos P, Lee K, et al. Paucity of dendritic cells in pancreatic cancer. Surgery 2002; 131: 135-8.

25. Iwamoto $M$, Shinohara $H$, Miyamoto $A$, et al. Prognostic value of tumor-infiltrating dendritic cells expressing CD83 in human breast carcinomas. Int J Cancer 2003; 104: 92-7.

26. Scarlett UK, Rutkowski MR, Rauwerdink AM, et al. Ovarian cancer progression is controlled by phenotypic changes in dendritic cells. J Exp Med 2012; 209: 495-506.

27. König S, Nitzki F, Uhmann A, et al. Depletion of cutaneous macrophages and dendritic cells promotes growth of basal cell carcinoma in mice. PLoS One 2014; 9: e93555.

28. Datta J, Berk E, Cintolo JA, Xu S, Roses RE, Czerniecki BJ. Rationale for a multimodality strategy to enhance the efficacy of dendritic cell-based cancer immunotherapy. Front Immunol 2015; 6: 271-81.

29. Van Heerden WFP, Raubenheimer EJ, van Rensburg EJ, le Roux R. Lack of correlation between DNA ploidy, Langerhans cell population and grading in oral squamous cell carcinoma. J Oral Pathol Med 1995; 24: 61-5.

30. O'Donnell RK, Mick R, Feldman M, et al. Distribution of dendritic cell subtypes in primary oral squamous cell carcinoma is inconsistent with a functional response. Cancer Lett 2007; 255: 145-52.

31. Kikuchi K, Kusama K, Sano M, et al. Vascular endothelial growth factor and dendritic cells in human squamous cell carcinoma of the oral cavity. Anticancer Res 2006; 26: 1833-48.

32. Seité $S$, Zucchi $H$, Moyal $D$, et al. Alterations in human epidermal Langerhans cells by ultraviolet radiation: quantitative and morphological study. Br J Dermatol 2003; 148: 291-9.

33. D'Errico M, Lemma T, Calcagnile A. Proietti De Santis L, Dogliotti E. Cell type and DNA damage specific response of human skin cells to environmental agents. Mutat Res 2007; 614: 37-47.

34. Jinlian L, Yingbin Z, Chunbo W. p38 MAPK in regulating cellular responses to ultraviolet radiation. J Biomed Sci 2007; 14: 303-12.

35. Beissert S, Loser K. Molecular and cellular mechanisms of photocarcinogenesis. Photochem Photobiol 2008; 84: 29-34. 
36. Furio L, Berthier-Vergnes O, Ducarre B, Schmitt D, Peguet-Navarro J. UVA radiation impairs phenotypic and functional maturation of human dermal dendritic cells. J Invest Dermatol 2005; 125: 1032-8.

37. Dumay O, Karam A, Vian L, et al. Ultraviolet Al exposure of human skin results in Langerhans cell depletion and reduction of epidermal antigen-presenting cell function: partial protection by a broadspectrum sunscreen. Br J Dermatol 2001; 144: 1161-8.

38. Araújo CP, Gurgel CA, Ramos EA, et al. Accumulation of CD1a-positive Langerhans cells and mast cells in actinic cheilitis. J Mol Histol 2010; 41: 357-65.

39. Warnakulasuriya S, Reibel J, Bouquot J, Dabelsteen E. Oral epithelial dysplasia classification systems: predictive value, utility, weakness and scope for improvement. J Oral Pathol Med 2008; 37: 127-33.

40. Carvalho MV, Pereira JS, Alves PM, Silveira EJD, Souza LB, Queiroz LMG. Alterations in the immunoexpression of galectins-1, -3 and -7 between different grades of oral epithelial dysplasia. $J$ Oral Pathol Med 2013; 42: 174-9. 